\title{
Naturally Derived Membrane Lipids Impact Nanoparticle-Based Messenger RNA Delivery
}

\author{
Jeonghwan Kim, ${ }^{1}$ Antony Jozic, ${ }^{1}$ and Gaurav Sahay (D) ${ }^{1,2}$ \\ ${ }^{1}$ Department of Pharmaceutical Sciences, College of Pharmacy, Oregon State University, Robertson Life Sciences Building, \\ Portland, OR, USA; and ${ }^{2}$ Department of Biomedical Engineering, Oregon Health Science University, Robertson Life Sciences \\ Building, Portland, OR, USA
}

(Received 17 February 2020; accepted 16 May 2020; published online 26 May 2020)

Associate Editor Michael R. King oversaw the review of this article

\begin{abstract}
Introduction-Lipid based nanoparticles (LNPs) are clinically successful vectors for hepatic delivery of nucleic acids. These systems are being developed for non-hepatic delivery of mRNA for the treatment of diseases like cystic fibrosis or retinal degeneration as well as infectious diseases. Localized delivery to the lungs requires aerosolization. We hypothesized that structural lipids within LNPs would provide features of integrity which can be tuned for attributes required for efficient hepatic and non-hepatic gene delivery. Herein, we explored whether naturally occurring lipids that originate from the cell membrane of plants and microorganisms enhance mRNA-based gene transfection in vitro and in vivo and whether they assist in maintaining mRNA activity after nebulization.

Methods - We substituted DSPC, a structural lipid used in a conventional LNP formulation, to a series of naturally occurring membrane lipids. We measured the effect of these membrane lipids on size, encapsulation efficiency and their impact on transfection efficiency. We further characterized LNPs after nebulization and measured whether they retained their transfection efficiency.

Results - One plant-derived structural lipid, DGTS, led to a significant improvement in liver transfection of mRNA. DGTS LNPs had similar transfection ability when administered in the nasal cavity to conventional LNPs. In contrast, we found that DGTS LNPs had reduced transfection efficiency in cells pre-and post-nebulization while maintaining size and encapsulation similar to DSPC LNPs.

Conclusions - We found that structural lipids provide differential mRNA-based activities in vitro and in vivo which also depend on the mode of administration. Understanding influence of structural lipids on nanoparticle morphology
\end{abstract}

Address correspondence to Gaurav Sahay, Department of Pharmaceutical Sciences, College of Pharmacy, Oregon State University, Robertson Life Sciences Building, Portland, OR, USA. Electronic mail: sahay@ohsu.edu and structure can lead to engineering potent materials for mRNA-based gene therapy applications.

Keywords-Lipid nanoparticles, Structural lipid, Gene delivery.

\begin{tabular}{|c|c|}
\hline & ABBREVIATIONS \\
\hline DSPC & $\begin{array}{l}\text { 1,2-Distearoyl-sn-glycero-3-phospho- } \\
\text { choline }\end{array}$ \\
\hline DGTS & $\begin{array}{l}\text { 1,2-Dipalmitoyl-sn-glycero-3-O-4'- } \\
\text { (N,N,N-trimethyl)-homoserine }\end{array}$ \\
\hline MGDG & Monogalactosyldiacylglycerol \\
\hline GDG & Digalactosyldiacylglycerol \\
\hline QDG & Sulfoquinovosyldiacylglycerol \\
\hline Cyclo PC & $\begin{array}{l}\text { 1-Palmitoyl-2-cis-9,10-methylenehexade- } \\
\text { canoyl-sn-glycero-3-phosphocholine }\end{array}$ \\
\hline & Polydispersity index \\
\hline & Lipid-based nanoparticles \\
\hline$o E$ & Apolipoprotein E \\
\hline & Polyethylene glycol \\
\hline
\end{tabular}

\section{INTRODUCTION}

The FDA approval of ONPATTRO ${ }^{\circledR}$, the first RNAi-based drug, has forged the pathway for next generation RNA-based therapeutics reaching the clinic. $^{3}$ It was enabled by the development of lipidbased nanoparticles (LNPs) that are used to deliver siRNA to hepatocytes preferentially. ${ }^{2}$ This technology has received wide interest and is being deployed to deliver larger nucleic acids, such as messenger RNA and genome-editing components. ${ }^{35,51}$ LNP-mediated 
intracellular delivery of nucleic acids occurs through its interactions with serum proteins, specifically apolipoprotein E (ApoE), which assists their cellular entry to hepatocytes via receptor-mediated endocytosis. $^{2,31}$ While LNPs enter hepatocytes in a highly efficient manner, effective cytosolic delivery of nucleic acids remains challenging. In particular, it has been consistently shown that most LNPs are sequestered and degraded in lysosomes ${ }^{12}$ or removed to extracellular space, ${ }^{44}$ significantly limiting their therapeutic effects. ${ }^{19,34}$ In addition, it is difficult to target nonhepatic organs with LNPs due to high liver tropism. It was demonstrated that spleen ${ }^{21,25}$ and lung endothelium $^{28,29}$ can be targeted by modification of LNP formulations. Nonetheless, the use of LNPs with regards to non-hepatic delivery is still limited, and is the area of focus for next generation therapeutics.

Typically, LNPs consist of four lipids: (1) cationic lipid, (2) PEG lipid, (3) cholesterol, and (4) structural lipid. The first component i.e. the cationic lipid is generally composed of a cationic head group and lipid tails, and is located in the core of LNPs with nucleic acids. This component is extensively explored because of its ability to assemble negatively charged nucleic acids into particles via electrostatic forces, and also in causing cytosolic release of nucleic acid by its interactions with the endosomal membrane. ${ }^{1,10,16,45}$ The second component is the PEG lipid which possesses a PEG chain anchored to a lipid headgroup. This molecule prevents aggregation and provides stability by causing steric hindrance on the LNP surface. ${ }^{31}$ Varying PEG lipid concentrations in LNPs is known to alter LNP size and transfection efficiency. ${ }^{31,50}$ Once injected, PEG lipid can disassemble in the serum, followed by ApoE binding and internalization. The third component is cholesterol which is involved in the intracellular delivery of LNPs. Absence of cholesterol receptors that recycle it from the endosomes can enhance the accumulation of LNPs, and thus increase the level of gene silencing. ${ }^{44}$ Arteta et al. stated that LNP surface may include cholesterol crystals due to its limited solubility and this surface configuration of LNPs may promote endosomal escape. ${ }^{50}$ Recent studies revealed that liver microenvironment other than hepatocytes can be targeted with LNPs containing oxidized cholesterols. ${ }^{38}$ We have reported that plant-based cholesterol analogs transformed LNP morphology and stimulated endosomal escape of LNPs. ${ }^{36}$ Finally, LNPs contain structural lipid which is primarily present on their surface. ${ }^{23,48}$ As the name suggests, it contributes to nucleic acid packaging and stability of the nanoparticles. ${ }^{22}$ Moreover, literature has highlighted that these helper or structural lipids enable structural transition of LNPs in endosomes, stimulating cytosolic release of nucleic acid. ${ }^{30,47} \mathrm{Li}$ et al. and Kauffman et al. compared 1,2-dioleoyl-snglycero-3-phosphoethanolamine (DOPE) and 1,2-distearoyl-sn-glycero-3-phosphocholine (DSPC) as structural lipids, and found that LNPs containing DOPE instead of DSPC more effectively delivered mRNA. ${ }^{18,24}$ It may suggest that the most effective LNP for mRNA delivery can be distinct from the current standard formulation which contains DSPC. Nonetheless, structural lipids for mRNA delivery have been less explored.

Herein, we studied naturally occurring lipids that originate from cell membrane of plants and microorganisms. These lipids carry the chemical and biological diversity associated with various aspects in biological membrane, such as membrane fluidity, permeability, and cell signaling. ${ }^{15}$ For these reasons, we adopted these membrane lipids to explore changes in LNP characteristics and mRNA transfection. Furthermore, our interests in local delivery to the lungs led us to use structural lipids for development of an inhalable form of LNPs. We examined whether structural lipids protect mRNA after nebulization for its local delivery to the lungs, and whether the diversity generated due to structural lipids improves LNP efficacy in terms of in vivo mRNA transfection. We found that certain plant-based membrane lipids can have differential transfection efficiency when delivered to cells, different organs or through different methods of administration.

\section{RESULTS}

In order to investigate the effects of structural lipid in LNP formulations, six different membrane lipids (DSPC, SQDG, MGDG, DGDG, Cyclo PC, and DGTS) were used. Among these structural lipids, DSPC served as a standard lipid since it is a widelyused phosphatidylcholine derivative within LNPs $^{10,35,40}$ and liposomes. ${ }^{33}$ MGDG, DGDG and SQDG are glycolipids that possess sugar moieties in their head groups. Glycolipids are not only a major constituent of structural lipids in microorganisms and plants, but are also responsible for various cell functions, such as signaling, cell recognition, and cell adhesion. ${ }^{41}$ Specifically, lipids containing a galactosylated head group provide lipid rafts with different properties including membrane fluidity and repulsion. $^{42}$ We focused on the roles of glycolipids in microorganisms, expecting incorporation of glycolipids to change the behaviors of LNPs. MGDG and DGDG have one or two galactoses, respectively; and their tails are composed of two unsaturated tails $(\alpha-$ linolenic acid, 18:3). SQDG incorporates a sulfoquinovosyl head group, and its tails are composed of one saturated tail (palmitic acid, C16) and one unsat- 
urated tail ( $\alpha$-linolenic acid, 18:3). DGTS is a betaine lipid and has shorter lipid tails (palmitic acid, C16) than DSPC (stearic acid, C18). DGTS is zwitterionic, much like DSPC, however it is phosphate-free. In addition, the configuration of zwitterionic headgroup in relation to the central glycerol of DGTS is different from that of DSPC. The phosphate group of DSPC is directly attached to the glycerol moiety whereas the zwitterionic moiety of DGTS is connected to the glycerol moiety by a branch of hydrocarbons. This configuration of DGTS may alter the membrane rigidity when present in the LNPs and influence the intracellular delivery of nucleic acids. Cyclo PC contains the same head group as DSPC, but its tail consists of one saturated tail (palmitic acid, C16) and one cyclic fatty acid (cis-9,10-methylenehexadecanoic acid). The unconventional and asymmetric tails in Cyclo PC may change the LNP stability by perturbing lipid packing. ${ }^{39}$ While we varied structural lipids, DLinMC3-DMA and DMG-PEG ${ }_{2 \mathrm{~K}}$ were used as the cationic lipid and PEG lipid, respectively (Fig. 1a). We used an optimized molar ratio from previous studies (DLin-MC3-DMA/Cholesterol/structural lipids/PEG Lipid $=50 / 38.5 / 10 / 1.5)$, and all lipids were prepared in pure ethanol, followed by microfluidic mixing with aqueous mRNA solution at 1:3 ratio (Fig. 1b). We characterized hydrodynamic sizes of LNPs using dynamic light scattering, and all LNPs prepared with different structural lipids exhibited $100-130 \mathrm{~nm}$ in diameters and narrow distributions (PDI $<0.2$ ) (Fig. 2a). The LNPs containing SQDG, MGDG, or DGDG showed 10-20 nm larger sizes than the DSPC LNPs. However, the Cyclo PC or DGTS LNPs had comparable sizes to the DSPC LNPs. Slight changes in the hydrodynamic size of the LNPs containing SQDG, MGDG, or DGDG was presumed to be due to their relatively bulky sugar moieties. In RNA encapsulation assay, all LNPs encapsulated more than $90 \%$ of mRNA (Fig. 2b). Afterward, we evaluated in vitro mRNA transfection in human epithelial HeLa cell line by overnight treatment with various LNP formulations encapsulating firefly luciferase mRNA. In overnight transfection, none of the LNP formulations inhibited the cell viability (Fig. 2c). For quantitative analysis of luciferase expression, we normalized bioluminescence by cell viability, and compared the results to those of DSPC LNPs. Between various LNP formulations, only Cyclo PC LNPs effectively transfected the cells compared to DSPC LNPs. It suggests that its structural differences to DSPC were not significant enough to influence in vitro transfection (Fig. 2d), which was demonstrated in the literature reporting that alteration of symmetrical lipid tails to asymmetrical tails did not significantly change the assembly and transfection of LNPs. ${ }^{46}$ DGTS LNPs produced 20 -fold lower lucifer- ase expression when compared to DSPC. Given the differences in chemical structure of the two lipids discussed above, such a significant difference in vitro transfection was unexpected. We studied if the two zwitterionic lipids endowed LNPs with different surface charges, but both LNPs exhibited similar zetapotentials: DSPC and DGTS LNPs showed $-5.6 \pm 3.4$ and $-4.9 \pm 0.8 \mathrm{mV}$, respectively. Dosedependent transfection studies between DSPC and DGTS LNPs highlighted that structural lipids play a role in mRNA transfection. Considering that structural lipids took only $10 \%$ of total lipids, the drastic differences in vitro transfection demonstrate the significant impact of structural lipids in LNPs. DSPC and DGTS LNPs both had no significant effect in cell viability at every dose tested (Fig. 1e). In the luciferase assay, results of the DGTS group were significantly weaker than those of the DSPC group. DSPC LNPs produced almost 40-fold higher luciferase expression when compared to DGTS LNPs at $200 \mathrm{ng}$ mRNA per well dose (Fig. 1f).

After evaluating in vitro transfection efficiency of LNP formulations, we investigated whether this drastic change in mRNA transfection would be reproducible in vivo. We focused primarily on DGTS since it has a zwitterionic nature similar to DSPC, which will retain the surface charge as of DSPC, and therefore it has more comparable structure that can be tested further. ${ }^{29}$ We intravenously administered DSPC or DGTS LNPs encapsulating firefly luciferase mRNA to $\mathrm{BALB} / \mathrm{c}$ mice, and monitored luminescent signals using in vivo imaging system (IVIS). Primary luciferase signals were detected in the liver as has been previously observed through a ApoE-dependent LNP uptake into hepatocytes. Interestingly, strong liver transfections were detected not only in the DSPC LNP-treated animals, but also in DGTS LNP-treated animals, from 4 to $48 \mathrm{~h}$ post-administration (Fig. 3a). In opposition to the results from in vitro transfection studies, DGTS LNPs resulted in improvement in luciferase expression when compared to DSPC LNPs in all time points (Fig. 3a). Images obtained from IVIS analysis were further analyzed to compare bioluminescent signals in the same regions of interest. In all time points for imaging, DGTS LNPs produced 4-fold stronger bioluminescent signals than DSPC LNPs $(p<0.05$, Fig. $3 b)$. These results indicated that LNP-mediated in vivo transfection is poorly correlated with in vitro transfection results, as demonstrated earlier., ${ }^{9,37}$ In addition, it implied that inclusion of different structural lipids affects interactions between nanoparticles and biological systems.

Having observed that DGTS LNPs effectively transfected liver, we investigated if DGTS LNPs would deliver mRNA efficiently to the respiratory system as 
(a)

DSPC

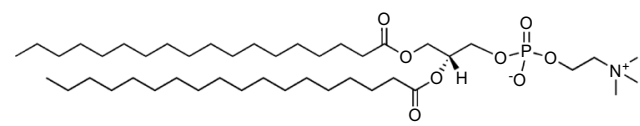

\section{Cyclo PC}

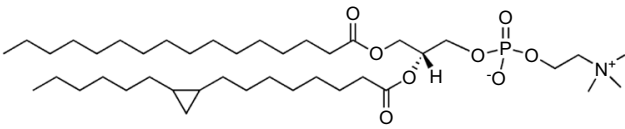

DGTS

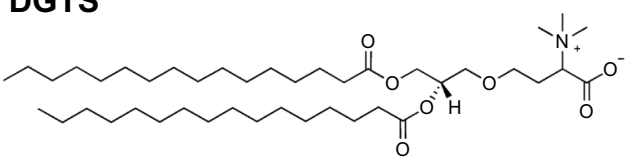

DLin-MC3-DMA

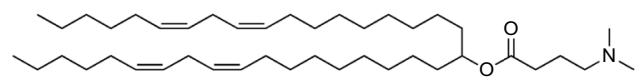

DMG-PEG ${ }_{2 K}$<smiles>CCCCCCCCCCCCCCCCCCC(=O)OCC(COCC(C)(C)COC(C)(C)COC)OC(=O)CCCCCCCCC</smiles>

\section{Cholesterol}

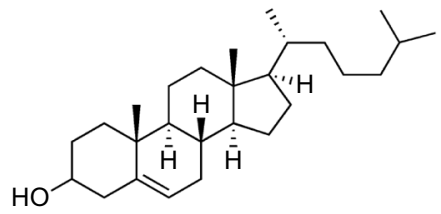

\section{MGDG}

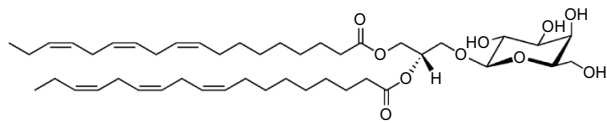

DGDG

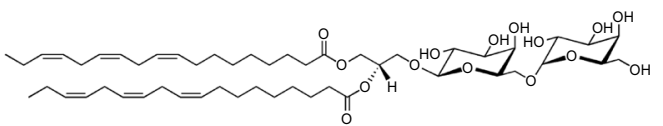

\section{SQDG}
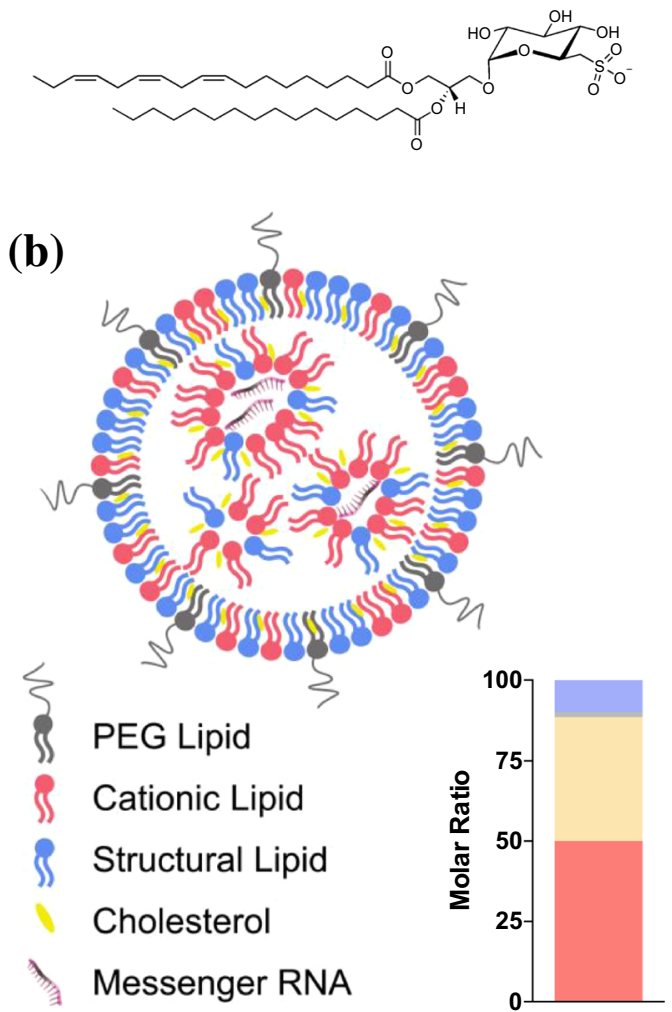

FIGURE 1. (a) Structures of naturally occurring membrane lipids, cationic lipid (DLin-MC3-DMA), PEG lipid (DMG-PEG 2 K), and cholesterol. (b) Schematic representation of LNP encapsulating mRNA. Inset, molar ratio of each lipid in the LNPs. Red; cationic lipid, sand; cholesterol, blue; structural lipids, gray; PEG lipid.

well. Delivery of mRNA to the respiratory system is highly desired for treatment of genetic and infectious diseases in the lungs. ${ }^{40}$ For delivery to pulmonary organs, nebulization, which generates aerosol from solution, is a non-invasive method of administration. Nonetheless, aerosolization of LNPs is considered challenging due to destabilization of LNPs during nebulization. We hypothesized that DGTS may affect physicochemical properties of LNPs, assisting mRNA transfection in lung epithelium after nebulization. We first studied changes in LNP size after nebulization using two nebulizer units that generate vapors with different sizes: one (standard, STD) aerosolizes LNP solutions to 4-6 $\mu \mathrm{m}$ diameter and the other (small, SML) aerosolizes LNP solutions to 2.5-4 $\mu \mathrm{m}$ diameter. Hydrodynamic size of DSPC and DGTS LNPs both increased to around $170 \mathrm{~nm}$, and PdI of both LNPs increased to 0.25 as well (Figs. $4 a$ and $4 b$ ). Size of DGTS LNPs were smaller than that of DSPC LNPs before nebulization, however it became similar post nebulization (Fig. 4a). In addition, size and PdI of LNPs were not affected by type of nebulization units (Figs. $4 \mathrm{a}$ and $4 \mathrm{~b}$ ). For in vitro mRNA transfection assay, we tested LNPs in A549 human lung epithelial 
(a)

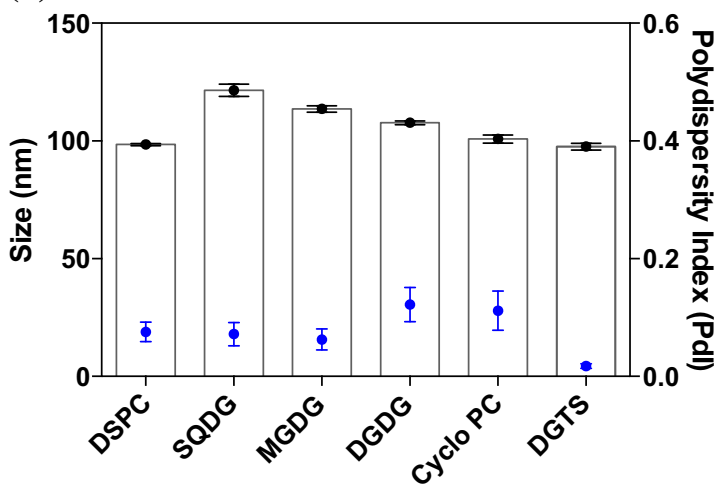

Structural Lipids

- $\quad$ Size $(\mathrm{nm})$

(c)

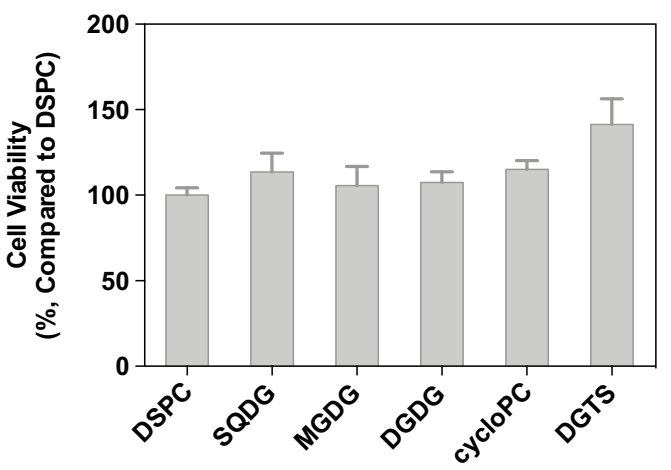

Structural Lipids

(e)

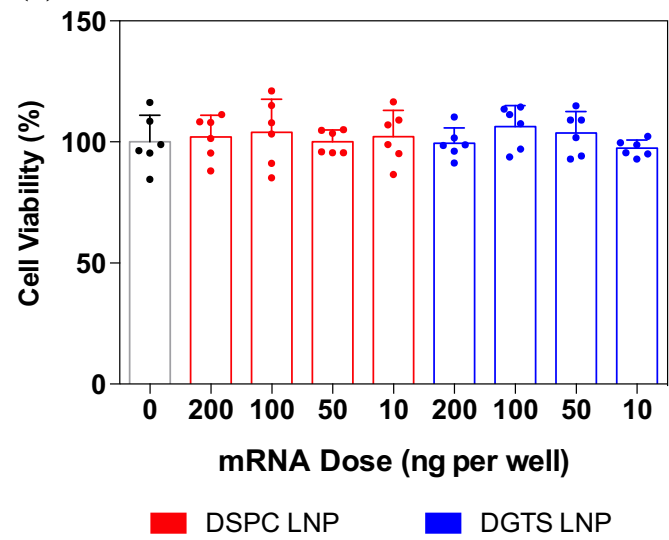

(b)

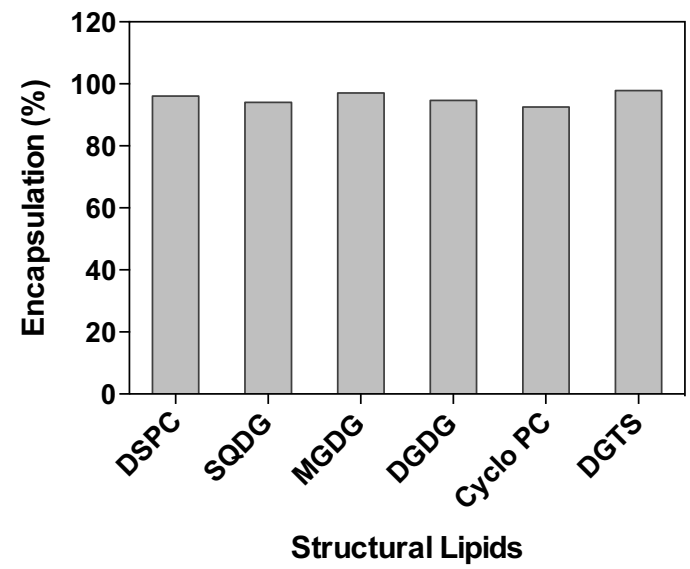

(d)

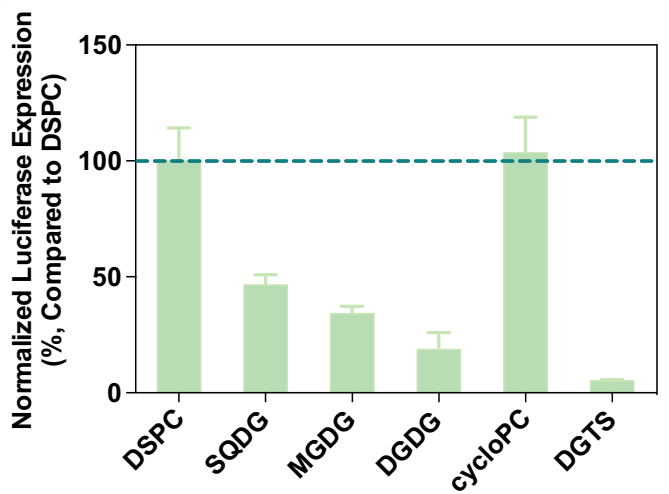

Structural Lipids

(f)

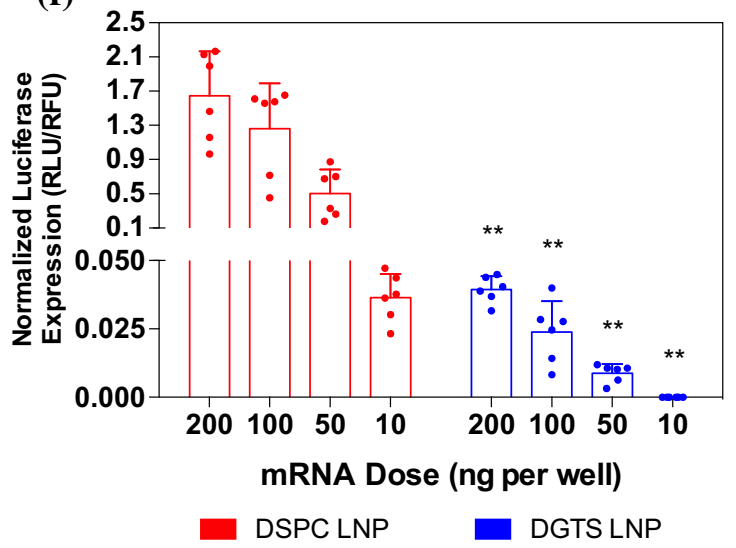

FIGURE 2. Characterizations of LNPs containing various structural lipids. (a) DLS analysis of LNPs containing various structural lipids $(n=3)$. Black circles; hydrodynamic size, Blue circles; Pdl (b) Encapsulation efficiency of LNPs containing various structural lipids. (c) Cell viability of HeLa after overnight treatment of various LNPs (50 ng mRNA/well, $n=3-8)$. (d) Relative luciferase expression in HeLa cells after overnight treatment of LNPs containing various structural lipids. Horizontal dashed line represents DSPC LNP expression set to 100 percent. (e) Cell viability and (f) Luciferase assay of HeLa cells transfected with DSPC or DGTS LNPs for $24 \mathrm{~h}(10-200 \mathrm{ng} \mathrm{mRNA} /$ well, $n=6)$. Comparisons between groups were performed in each dose using Student's $t$ test. ${ }^{* *} p<0.01$. All data were expressed as the mean \pm S.D.

cell line. When A549 cells were treated with the LNPs in various mRNA doses, no inhibition in cell viability was observed (Fig. 4c). In vitro luciferase assay revealed that DSPC LNPs transfected A549 cells more effectively than DGTS LNPs in a dose-dependent manner, which corresponds to the results from $\mathrm{HeLa}$ cells. We also evaluated LNP-mediated mRNA transfection to A549 cells with nebulization. Nebulized 
(a)

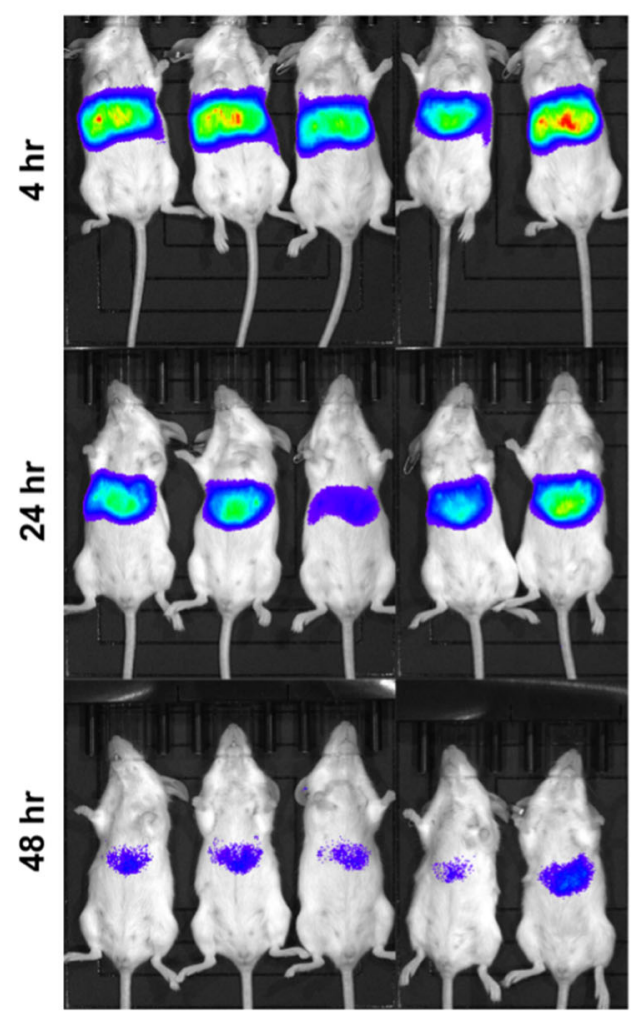

DSPC LNP

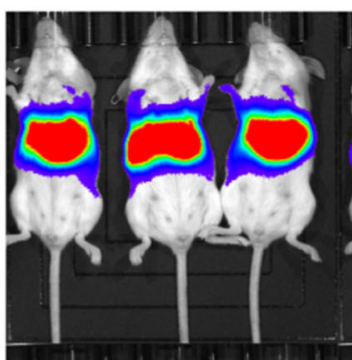

\section{DGTS LNP}

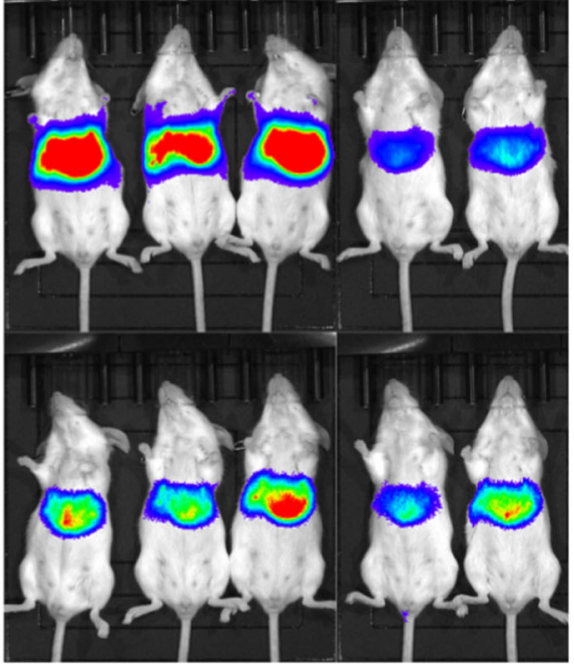

Luminescence

Radiance (p/sec/cm $/ 2 / \mathrm{sr}$ )

1.0

$-0.8$

$-0.6$

$\times 10^{8}$

$-0.4$

0.2 Colar Scale

Min $=4.00 \mathrm{e} 6$

$\operatorname{Max}=1.00 \mathrm{e} 8$

1.0

0.8

$0.6 \times 10^{7}$

0.4

Colar Scale

$0.2 \mathrm{Min}=1.00 \mathrm{e} 6$ $\operatorname{Max}=1.00 \mathrm{e} 7$

(b)

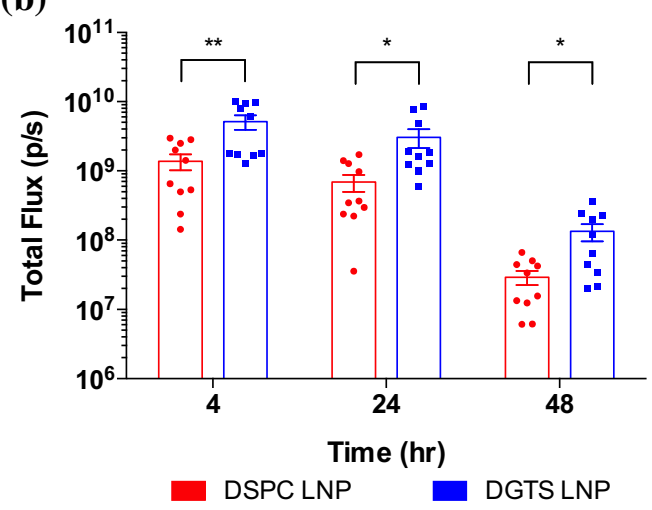

FIGURE 3. (a) Representative IVIS images of BALB/c mice after treatment of $0.1 \mathrm{mg} / \mathrm{kg}$ mRNA delivered through IV injection of DSPC or DGTS LNP $(n=5)$. (b) Quantification of luminescent signals from IVIS images. ROI kept constant in all images $(n=10)$. ${ }^{\star} p<0.05,{ }^{* *} p<0.01$, Student's $t$-test. All data were expressed as the mean \pm S.E.M.

LNPs didn't affect cell viability of A549 cells (Fig. 4e), and luciferase expression was higher in the cells treated with DSPC LNPs than DGTS LNPs (Fig. 4f).

In order to assess in vivo transfection of LNPs to epithelium, we examined the luciferase expression in nasal respiratory epithelium. Nasal respiratory epithelium was chosen to evaluate the transfection of epithelial cells because it is not only an easily accessible epithelium with local administrations, but also physiologically similar to lung epithelium, supported by the fact that clinical diagnosis of lung diseases like cystic fibrosis is performed by monitoring nasal potential difference in nasal epithelium. ${ }^{40,43} \mathrm{We}$ intranasally instilled LNPs into nostrils of animals to transfect the nasal epithelium, and luciferase expressions were imaged using IVIS at 4 and $24 \mathrm{~h}$ after the administration. All animals displayed luminescent signals around the nasal cavity in $4 \mathrm{~h}$, and the signals intensified $24 \mathrm{~h}$ after the administration (Fig. 5a). In $4 \mathrm{~h}$ after the treatment, both DSPC $\left(10.6 \times 10^{5}\right.$ photon/s $)$ 
(a)

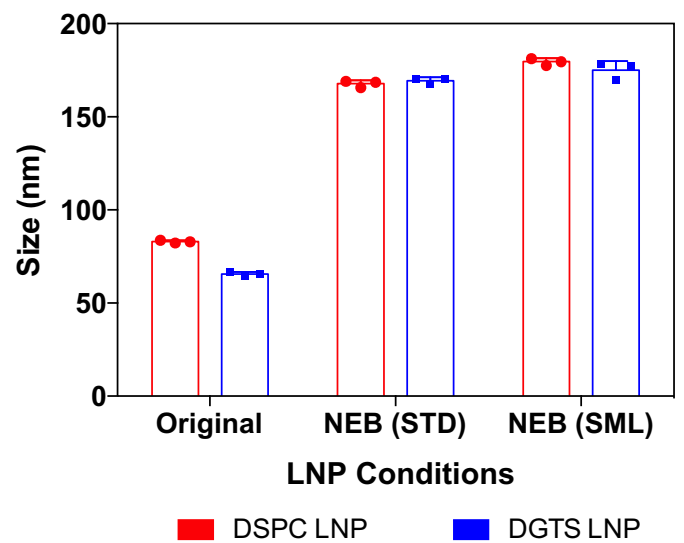

(c)

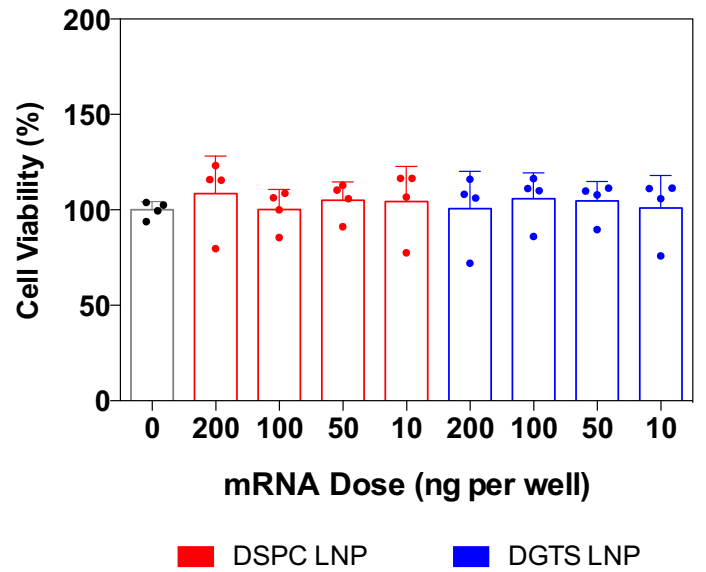

(e)

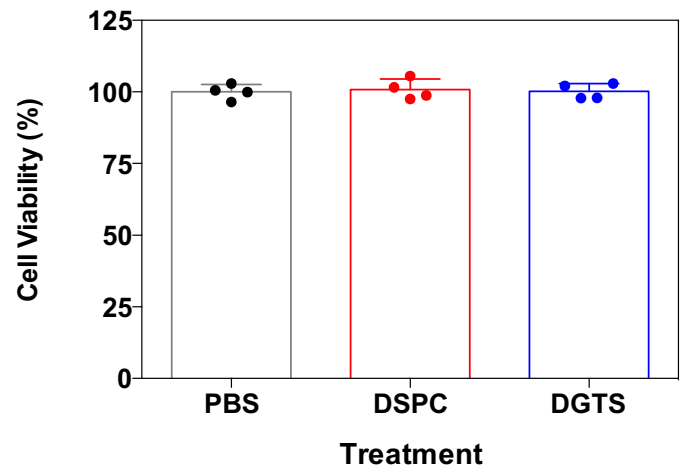

(b)

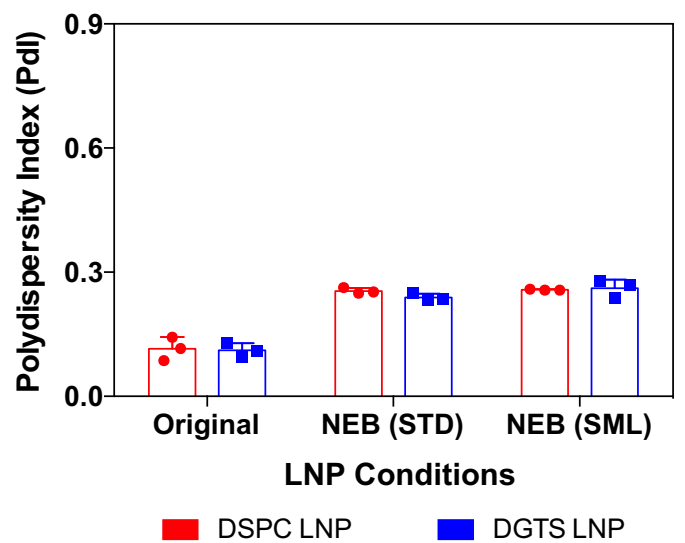

(d)

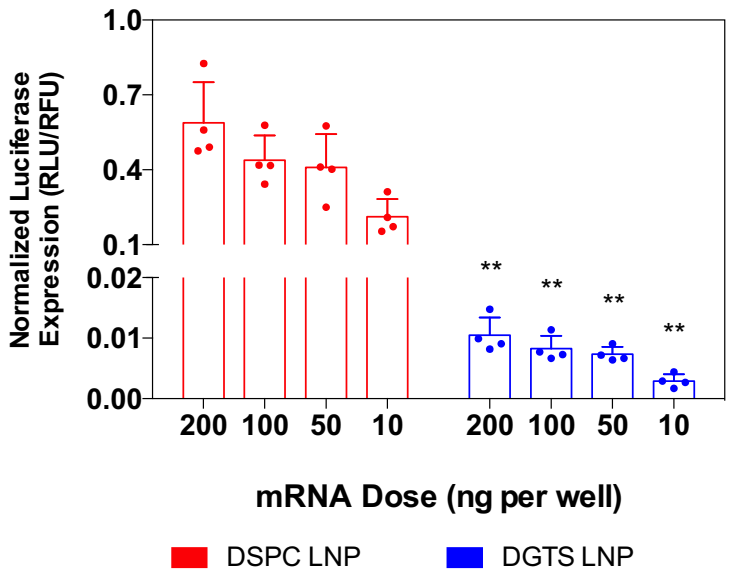

(f)

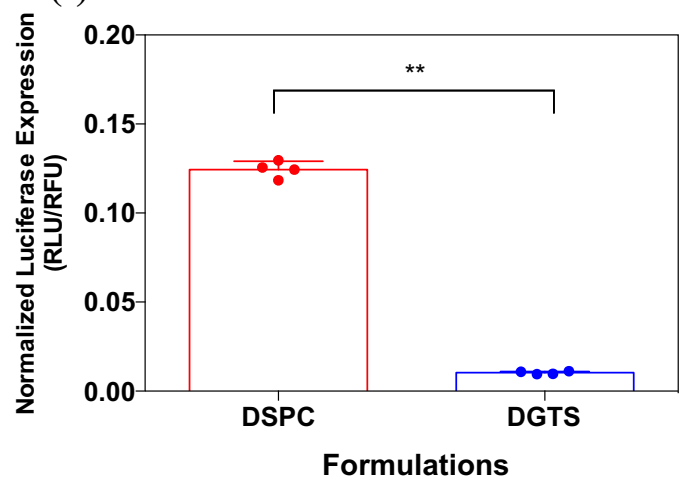

FIGURE 4. Characterizations of DSPC and DGTS LNPs with or without nebulization. (a) Size and (b) Pdl of original and nebulized (NEB) LNPs containing DSPC or DGTS $(n=3)$. STD; a standard nebulizer unit (vapor median diameter $=4-6 \mu \mathrm{m}$ ), SML; a small nebulizer unit (vapor median diameter $=2.5-4 \mu \mathrm{m}$ ) (c) Cell viability and (d) Luciferase expression of A549 cells transfected with DSPC or DGTS LNPs for $24 \mathrm{~h}$ in a 96 well plate (10-200 ng mRNA/well, $n=4)$. (e) Cell viability and (f) Luciferase expression of A549 cells transfected with nebulized DSPC or DGTS LNPs for $24 \mathrm{~h}$ in a 12 well plate $(1 \mu \mathrm{g} \mathrm{mRNA} / \mathrm{well}, n=4)$. Comparisons between groups were performed in each dose using Student's t-test. ${ }^{* \star} p<0.01$. All data were expressed as the mean \pm S.D.

and DGTS LNPs $\left(7.6 \times 10^{5}\right.$ photon/s) exhibited similar luciferase activity in animals $(p=0.18$, Fig. $5 b)$; however, $24 \mathrm{~h}$ after the treatment, luciferase expres- sions of DSPC LNP-treated animals $\left(5.52 \times 10^{5}\right.$ photon/s) were higher than those of DGTS LNP-treated animals $\left(2.60 \times 10^{5}\right.$ photons/s, $p<0.01$, Fig. $\left.5 b\right)$. It 


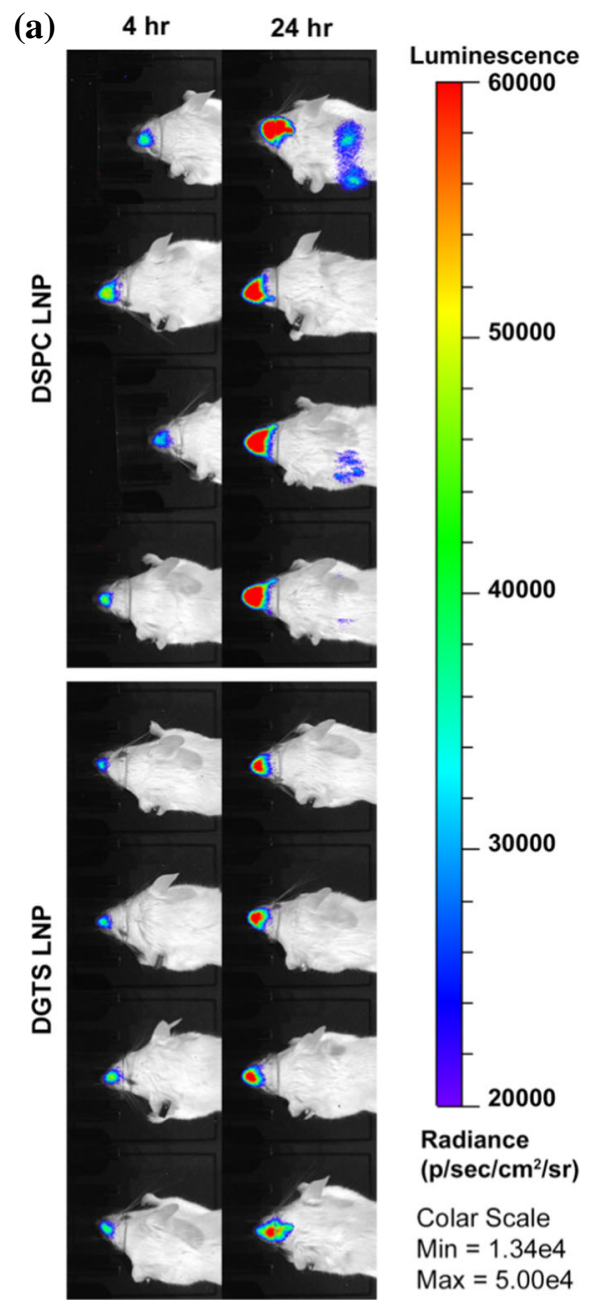

(b)

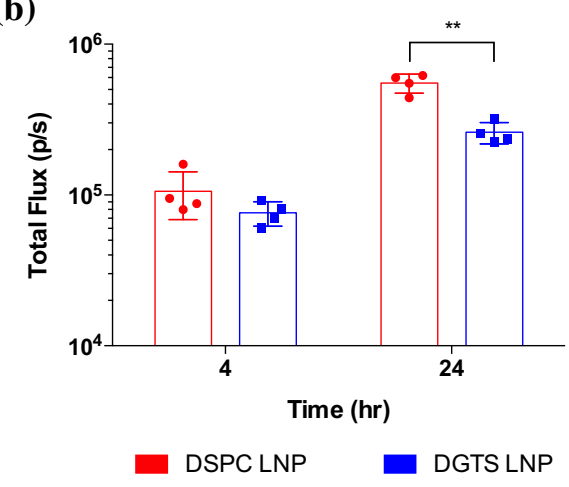

FIGURE 5. (a) Transfection of BALB/c mice treated with $0.25 \mathrm{mg} / \mathrm{kg}$ mRNA delivered through intranasal instillation of DSPC or DGTS LNP $(n=4)$. (b) Quantification of luminescent signals from IVIS images. ROI kept constant in all images $(n=4) .{ }^{* \star} p<0.01$, Student's t-test. All data were expressed as the mean \pm S.D.

was also notable that some animals treated with DSPC LNPs exhibited mild lung transfection, suggesting that a portion of administered LNPs flowed into the airway and transfected lung epithelium (Fig. 5a). These results demonstrated that DSPC LNPs transfect nasal epithelium more effectively than DGTS LNPs, which is opposed to the results of liver transfection. We are currently testing the ability of nebulized nanoparticles containing structural variants and their impact on gene delivery when delivered locally to the airway in vivo.

\section{DISCUSSION}

Naturally occurring lipids are present in living organisms and possess greater structural diversity compared to synthetic lipids. ${ }^{14}$ While we have accumulated insights into the lipid biosynthesis and metabolism in many species, it is still relatively unknown how these diverse lipids engage with subcellular mechanisms and signaling. The five bioactive lipids studied here are found in plant or bacterial cells which contain lipid compositions different from mammalian cells. Glycoglycerolipids, MGDG, DGDG, and SQDG, can be found in cellular organelles, more precisely in chloroplast of plant cells, and travel from the endoplasmic reticulum to various organelles for biosynthesis. ${ }^{5}$ DGTS is widely found in algae, and is thought to be associated with lipid metabolism ${ }^{49}$ and cell survival in stress conditions. ${ }^{20,32}$ Cyclo PC is found in the membrane of Escherichia coli. ${ }^{27}$ The reasons for species specific differences within this class of lipid remain unclear but these bioactive lipids have unexpected effects when incubated with mammalian cells, for example anti-inflammatory response. ${ }^{4,6}$

We have explored naturally occurring lipids to improve intracellular delivery of nucleic acids and demonstrated that cholesterol analogs identified in natural products boost endosomal escape of LNPs. ${ }^{36}$ We then wanted to understand whether naturally occurring structural lipids can lead to a better packing within the LNPs, uptake and/or amenable release of cargo inside cells. Traditionally, the role of structural lipid is acknowledged as providing encapsulation and stability and endosomal escape, ${ }^{10}$ but the diversity of structural lipid has been less explored than other components to reveal differences in transfection efficiency. Recent studies revealed that LNP surface contains PEG lipid, structural lipid and cholesterol, while the LNP core contains the cationic lipid and cargo ${ }^{48,50}$ Considering that PEG lipids is shed off after exposure to in vivo environment, LNP surface therefore is enriched with structural lipid and cholesterol. ${ }^{50}$ Since structural lipid and cholesterol are also known to assist cationic lipid-mediated destabilization of the endosomal membrane, ${ }^{36,47}$ any structural change caused by naturally occurring lipids could be critical in intracellular delivery. 
In this study, we were able to show that these lipids influence LNP efficacy without compromising size and RNA encapsulation, indicating their impact in intracellular delivery of mRNA. The incorporation of DGTS in LNP formulation resulted in variable efficacies depending on experimental conditions: in vitro cell culture, in vivo liver and epithelium. This discrepancy may be due to possible modification of LNP morphology by DGTS that changes the stability and tolerance of LNPs. ${ }^{8,30}$ It has already been argued that LNPs may require a reasonable compromise in stability for better transfection, suggesting that high stability is not beneficial for endosomal escape due to inhibition of membrane destabilization. ${ }^{8}$ Accordingly, stability of DGTS LNPs could be too high to transfect in vitro, but it could be beneficial for in vivo tolerability. One might also posit that structurally modified LNP surface potentially affects ApoE binding which may be the reason for this variability. ApoE is present at various concentrations depending on tissues. ${ }^{13,17}$ If DGTS facilitates the LNP binding to ApoE, DGTS LNPs will be internalized more efficiently in vivo and therefore we would find differential activity based on different tissues. Thus, structural lipids could be an important ingredient for developing tissue specificity. Upon further comparison of DGTS or DSPC as an excipient for inhalable LNPs, we found that the difference in in vitro mRNA transfection between DGTS and DSPC decreased after nebulization (about 50 folds vs. 12 folds), which probably implies advantage of DGTS in LNP tolerability for nebulization. Because loss of nanoparticles during the nebulization blocks delivering nucleic acids to the lungs via inhalation, ${ }^{11}$ discovery of novel structural lipids that make LNPs resilient to the use of nebulizer will substantially improve efficacy of gene therapies.

In summary, these studies reinforce the potential of structural lipids to target different organs. First, the presence of structural lipids at nanoparticle surface post PEG shedding makes them an important partner for serum proteins to form a corona. The composition of the protein corona on different nanoparticles is an active area of research. ${ }^{7,26}$ Ability to mitigate corona formation can enable LNPs to reach tissues beyond the liver. On the other hand, the surface can be designed to adsorb proteins which exploit processes that traverse impenetrable organs like the brain or propel the nanocarriers across lung endothelium. Second, the membrane lipids can play an important role in the formulation of nanoparticles based on routes of administration. For example, when delivered through inhalation, the structural lipid can impart a valuable feature to the nanocarrier, i.e., maintain its structure and ability to transfect after nebulization. Such fea- tures are necessary for developing inhalable mRNA nanoformulations for treatment of diseases like cystic fibrosis, COVID-19, asthma, or lung cancer that can be benefited by direct access to the lungs. Finally, it is unclear whether the membrane lipids directly engage with the process of endosomal escape. However, based on the differences within in vitro transfections with use of different structural lipids, one may speculate that the structural lipid perhaps contributes to LNP disassembly and resulting cytosolic delivery of RNA. The discovery of natural products that undergo conformational changes once inside an endosome can lead to potent nanocarriers for intracellular gene delivery.

\section{METHODS}

Materials

Firefly luciferase mRNA was purchased from Trilink Biotechnologies (L-7202). Cholesterol and DMG$\mathrm{PEG}_{2 \mathrm{~K}}$ were obtained from Sigma-Aldrich (MO, USA) and NOF America (CA, USA), respectively. DLin-MC3-DMA was custom synthesized by BioFine International Inc. (BC, Canada). All structural lipids including DSPC, DGDG, MGDG, SQDG, Cyclo PC, and DGTS, were purchased from Avanti Polar Lipids, Inc. (AL, USA).

\section{LNP Formulation and Characterization}

LNPs composed of DLin-MCD-DMA, Cholesterol, $\mathrm{DMG}_{-} \mathrm{PEG}_{2 \mathrm{~K}}$, structural lipids, and mRNA were prepared using microfluidic mixing as described previously. ${ }^{35}$ In brief, all lipids were dissolved in pure ethanol at 50:38.5:1.5:10 molar ratio, and mRNA was diluted in sterile $50 \mathrm{mM}$ citrate buffer. The lipid and mRNA solutions were mixed using the Nanoassemblr Benchtop at a 1:3 ratio, followed by 10-fold dilution with sterile PBS. Resulting LNP solutions were concentrated using Amicon ${ }^{\circledR}$ Ultra centrifugal filter units with 10,000 Da molecular-weight-cut-off (Millipore). Hydrodynamic size and PDI of the LNPs were analyzed in dynamic light scattering using a Zetasizer Nano ZSP (Malvern Instruments, UK). mRNA encapsulation was assayed using a Quant-iT ${ }^{\mathrm{TM}}$ RiboGreen ${ }^{\circledR}$ RNA Assay kit (Thermo) and a multimode microplate reader (Tecan Trading AG, Switzerland).

\section{Cell Culture}

HeLa and A549 cell lines were kindly gifted from Prof. Langer (MIT) and Prof. Alani (OSU), respectively. HeLa cells and A549 cells were cultured in DMEM and RPMI, respectively, supplemented with 
$10 \%$ heat-inactivated FBS and $1 \%$ penicillin/streptomycin.

\section{In Vitro mRNA Transfection Assay}

For in vitro mRNA transfection assays, cells were seeded on a white 96 well plate at $4 \times 10^{3}$ cells/well and allowed to attach overnight. Cells were treated with LNPs encapsulating FLuc mRNA for overnight to $24 \mathrm{~h}$ and analyzed for cell viability as well as luciferase expression with the ONE-Glo ${ }^{\mathrm{TM}}+$ Tox luciferase reporter and cell viability assay kit (Promega) using a multimode microplate reader.

\section{Animals}

All animal studied were conducted at Oregon Health and Sciences University and approved by the Institutional Animal Care and Use Committee (IACUC, IP00001707).

\section{In Vivo mRNA Transfection}

Female BALB/c mice (5-8 weeks) were anesthetized using isoflurane, and LNPs were administered to animals in various routes. For intravenous administration, LNPs encapsulating Fluc mRNA were injected into the mouse tail vein $(0.1 \mathrm{mg} / \mathrm{kg})$. For intranasal instillation, LNPs were pipetted onto a single nostril with spontaneous inhalation $(0.25 \mathrm{mg} / \mathrm{kg})$. At predetermined time points post-administration, $200 \mu \mathrm{l}$ of Dluciferin substrate was intraperitoneally injected to the mice $10 \mathrm{~min}$ prior to bioluminescent imaging $(150 \mathrm{mg}$ / $\mathrm{kg}$ ). Image acquisition and analysis were performed using the IVIS ${ }^{\circledR}$ Lumina XRMS and the manufacturer's software (PerkinElmer, MA).

\section{LNP Nebulization}

To nebulize LNPs, an Aeroneb ${ }^{\circledR}$ Lab Control Module equipped with a lab nebulizer unit (Aerogen, Ireland) was used. LNPs were added to the nebulizer unit for aerosolization, and the nebulized LNPs were collected for transfection and DLS analysis. For in vitro mRNA transfection, cells were seeded onto a 12 well plate at $5 \times 10^{4}$ cells/well and allowed to attach for overnight incubation. LNPs were diluted with serum-free media and nebulized with a small nebulizer unit onto the seeded cells $(1 \mu \mathrm{g} /$ well $)$, followed by $24-\mathrm{h}$ incubation. Cell viability and in vitro luciferase expression was assayed using ONE-Glo ${ }^{\mathrm{TM}}+$ Tox luciferase reporter and cell viability assay kit (Promega) and a multimode microplate reader.

\section{ACKNOWLEDGMENTS}

This project was supported through funding National Institute of Biomedical Imaging and Bioengineering (N.I.B.I.B) 1R15EB021581-01 (G.S), National Heart Lung and Blood Institute (N.H.L.B.I) 1R01HL146736-01 (G.S) and Cystic Fibrosis Foundation-SAHAY18G0 (G.S). We thank Ashwani Kumar, Anindit Mukherjee and Mohit Gupta for feedback.

\section{AUTHOR CONTRIBUTIONS}

G.S. conceived of the idea and directed the entire research. J.K. performed all the studies. A.J. helped J.K. in data acquisition. G.S., J.K. wrote the paper with feedback from A.J.

\section{CONFLICT OF INTEREST}

$\mathrm{JK}, \mathrm{AZ}$ and GS have no conflict of interest to report for this study.

\section{ETHICAL APPROVAL}

No human studies were carried out by the authors for this article. All animal studies were conducted at Oregon Health and Sciences University and approved by the Institutional Animal Care and Use Committee (IACUC, IP00001707).

\section{REFERENCES}

\footnotetext{
${ }^{1}$ Akinc, A., et al. A combinatorial library of lipid-like materials for delivery of RNAi therapeutics. Nat. Biotechnol. 26:561-569, 2008.

${ }^{2}$ Akinc, A., et al. Targeted delivery of RNAi therapeutics with endogenous and exogenous ligand-based mechanisms. Mol. Ther. 18:1357-1364, 2010.

${ }^{3}$ Akinc, A., et al. The Onpattro story and the clinical translation of nanomedicines containing nucleic acid-based drugs. Nat. Nanotechnol. 14:1084-1087, 2019.

${ }^{4}$ Banskota, A. H., R. Stefanova, S. Sperker, and P. J. McGinn. New diacylglyceryltrimethylhomoserines from the marine microalga Nannochloropsis granulata and their nitric oxide inhibitory activity. J. Appl. Phycol. 25:15131521, 2013.

${ }^{5}$ Benning, C. A role for lipid trafficking in chloroplast biogenesis. Prog. Lipid Res. 47:381-389, 2008.

${ }^{6}$ Bruno, A., et al. Selective in vivo anti-inflammatory action of the galactolipid monogalactosyldiacylglycerol. Eur. J. Pharmacol. 524:159-168, 2005.

${ }^{7}$ Cedervall, T., et al. Understanding the nanoparticle-protein corona using methods to quantify exchange rates and affinities of proteins for nanoparticles. Proc. Natl. Acad. Sci. 104:2050-2055, 2007.
} 
${ }^{8}$ Cheng, X., and R. J. Lee. The role of helper lipids in lipid nanoparticles (LNPs) designed for oligonucleotide delivery. Adv. Drug Deliv. Rev. 99:129-137, 2016.

${ }^{9}$ Cheng, Q., T. Wei, L. Farbiak, L. T. Johnson, S. A. Dilliard, and D. J. Siegwart. Selective organ targeting (SORT) nanoparticles for tissue-specific mRNA delivery and CRISPR-Cas gene editing. Nat. Nanotechnol. 15:313-320, 2020.

${ }^{10}$ Cullis, P. R., and M. J. Hope. Lipid nanoparticle systems for enabling gene therapies. Mol. Ther. 25:1467-1475, 2017.

${ }^{11}$ Elhissi, A., H. Gill, W. Ahmed, and K. Taylor. Vibratingmesh nebulization of liposomes generated using an ethanolbased proliposome technology. J. Liposome Res. 21:173180, 2011.

${ }^{12}$ Gilleron, J., et al. Image-based analysis of lipid nanoparticle-mediated siRNA delivery, intracellular trafficking and endosomal escape. Nat. Biotechnol. 31:638-646, 2013.

${ }^{13}$ Gordon, E. M., et al. Apolipoprotein E is a concentrationdependent pulmonary danger signal that activates the NLRP3 inflammasome and IL-1 $\beta$ secretion by bronchoalveolar fluid macrophages from asthmatic subjects. $J$. Allergy Clin. Immunol. 144:426-441.e3, 2019.

${ }^{14}$ Hong, J. Role of natural product diversity in chemical biology. Curr. Opin. Chem. Biol. 15:350-354, 2011.

${ }^{15}$ Ibarguren, M., D. J. López, and P. V. Escribá. The effect of natural and synthetic fatty acids on membrane structure, microdomain organization, cellular functions and human health. Biochim. Biophys. Acta BBA Biomembr. 15181528:2014, 1838.

${ }^{16}$ Jayaraman, M., et al. Maximizing the potency of sirna lipid nanoparticles for hepatic gene silencing in vivo. Angew. Chem. Int. Ed. 51:8529-8533, 2012.

${ }^{17}$ Jendresen, C., V. Årskog, M. R. Daws, and L. N. G. Nilsson. The Alzheimer's disease risk factors apolipoprotein E and TREM2 are linked in a receptor signaling pathway. J. Neuroinflammation 14:59, 2017.

${ }^{18}$ Kauffman, K. J., et al. Optimization of lipid nanoparticle formulations for mRNA delivery in vivo with fractional factorial and definitive screening designs. Nano Lett. 15:7300-7306, 2015.

${ }^{19}$ Kim, J., A. Narayana, S. Patel, and G. Sahay. Advances in intracellular delivery through supramolecular self-assembly of oligonucleotides and peptides. Theranostics 9:3191-3212, 2019.

${ }^{20}$ Kotlova, E. R., and N. F. Sinyutina. The role of betaine lipids in adaptation of lichen peltigera aphthosa to longterm dehydration. In: Advanced Research on Plant Lipids, edited by N. Murata, M. Yamada, I. Nishida, H. Okuyama, J. Sekiya, and W. Hajime. Dordrecht: Springer, 2003, pp. 373-376.

${ }^{21}$ Kranz, L. M., et al. Systemic RNA delivery to dendritic cells exploits antiviral defence for cancer immunotherapy. Nature 534:396-401, 2016.

${ }^{22}$ Kulkarni, J. A., D. Witzigmann, J. Leung, Y. Y. C. Tam, and P. R. Cullis. On the role of helper lipids in lipid nanoparticle formulations of siRNA. Nanoscale 11:2173321739, 2019.

${ }^{23}$ Kulkarni, J. A., et al. On the formation and morphology of lipid nanoparticles containing ionizable cationic lipids and siRNA. ACS Nano 12:4787-4795, 2018.

${ }^{24} \mathrm{Li}$, B., et al. An orthogonal array optimization of lipid-like nanoparticles for mRNA delivery in vivo. Nano Lett. 15:8099-8107, 2015.

${ }^{25}$ Lokugamage, M. P., C. D. Sago, Z. Gan, B. R. Krupczak, and J. E. Dahlman. Constrained nanoparticles deliver
siRNA and sgRNA to T cells in vivo without targeting ligands. Adv. Mater. 31:1902251, 2019.

${ }^{26}$ Lundqvist, M., J. Stigler, G. Elia, I. Lynch, T. Cedervall, and K. A. Dawson. Nanoparticle size and surface properties determine the protein corona with possible implications for biological impacts. Proc. Natl. Acad. Sci. 105:1426514270, 2008.

${ }^{27}$ Maric, S., et al. Biosynthetic preparation of selectively deuterated phosphatidylcholine in genetically modified Escherichia coli. Appl. Microbiol. Biotechnol. 99:241-254, 2015.

${ }^{28}$ Miller, J. B., P. Kos, V. Tieu, K. Zhou, and D. J. Siegwart. Development of cationic quaternary ammonium sulfonamide amino lipids for nucleic acid delivery. ACS Appl. Mater. Interfaces 10:2302-2311, 2018.

${ }^{29}$ Miller, J. B., et al. Non-viral CRISPR/Cas gene editing in vitro and in vivo enabled by synthetic nanoparticle codelivery of Cas 9 mRNA and sgRNA. Angew. Chem. Int. Ed. 56:1059-1063, 2017.

${ }^{30}$ Mochizuki, S., et al. The role of the helper lipid dioleoylphosphatidylethanolamine (DOPE) for DNA transfection cooperating with a cationic lipid bearing ethylenediamine. Biochim. Biophys. Acta BBA Biomembr. 412-418:2013, 1828.

${ }^{31}$ Mui, B. L., et al. Influence of polyethylene glycol lipid desorption rates on pharmacokinetics and pharmacodynamics of siRNA lipid nanoparticles. Mol. Ther. Nucleic Acids 2:e139, 2013.

${ }^{32}$ Murakami, H., T. Nobusawa, K. Hori, M. Shimojima, and H. Ohta. Betaine lipid is crucial for adapting to low temperature and phosphate deficiency in Nannochloropsis. Plant Physiol. 177:181-193, 2018.

${ }^{33}$ Papahadjopoulos, D., et al. Sterically stabilized liposomes: improvements in pharmacokinetics and antitumor therapeutic efficacy. Proc. Natl. Acad. Sci. 88:11460-11464, 1991.

${ }^{34}$ Patel, S., J. Kim, M. Herrera, A. Mukherjee, A. V. Kabanov, and G. Sahay. Brief update on endocytosis of nanomedicines. Adv. Drug Deliv. Rev. 144:90-111, 2019.

${ }^{35}$ Patel, S., et al. Boosting intracellular delivery of lipid nanoparticle-encapsulated mRNA. Nano Lett. 17:57115718, 2017.

${ }^{36}$ Patel, S., et al. Naturally-occurring cholesterol analogues in lipid nanoparticles induce polymorphic shape and enhance intracellular delivery of mRNA. Nat. Commun. 11:983, 2020.

${ }^{37}$ Paunovska, K., et al. A direct comparison of in vitro and in vivo nucleic acid delivery mediated by hundreds of nanoparticles reveals a weak correlation. Nano Lett. 18:2148-2157, 2018.

${ }^{38}$ Paunovska, K., et al. Nanoparticles containing oxidized cholesterol deliver mRNA to the liver microenvironment at clinically relevant doses. Adv. Mater. 31:1807748, 2019.

${ }^{39}$ Poger, D., and A. E. Mark. Effect of ring size in $\omega$-alicyclic fatty acids on the structural and dynamical properties associated with fluidity in lipid bilayers. Langmuir 31:11574-11582, 2015.

${ }^{40}$ Robinson, E., et al. Lipid nanoparticle-delivered chemically modified mRNA restores chloride secretion in cystic fibrosis. Mol. Ther. 26:2034-2046, 2018.

${ }^{41}$ Róg, T., and I. Vattulainen. Cholesterol, sphingolipids, and glycolipids: what do we know about their role in raft-like membranes? Chem. Phys. Lipids 184:82-104, 2014.

${ }^{42}$ Róg, T., I. Vattulainen, A. Bunker, and M. Karttunen. Glycolipid membranes through atomistic simulations: ef- 
fect of glucose and galactose head groups on lipid bilayer properties. J. Phys. Chem. B 111:10146-10154, 2007.

${ }^{43}$ Rowe, S. M., J. P. Clancy, and M. Wilschanski. Nasal potential difference measurements to assess CFTR ion channel activity. In: Cystic Fibrosis, edited by M. D. Amaral, and K. Kunzelmann. Totowa, NJ: Humana Press, 2011, pp. 69-86.

${ }^{44}$ Sahay, G., et al. Efficiency of siRNA delivery by lipid nanoparticles is limited by endocytic recycling. Nat. Biotechnol. 31:653-658, 2013.

${ }^{45}$ Semple, S. C., et al. Rational design of cationic lipids for siRNA delivery. Nat. Biotechnol. 28:172-176, 2010.

${ }^{46}$ Suzuki, Y., and H. Ishihara. Structure, activity and uptake mechanism of siRNA-lipid nanoparticles with an asymmetric ionizable lipid. Int. J. Pharm. 510:350-358, 2016.

${ }^{47}$ Tenchov, B. G., R. C. MacDonald, and D. P. Siegel. Cubic phases in phosphatidylcholine-cholesterol mixtures: cholesterol as membrane "fusogen". Biophys. J. 91:25082516, 2006.

${ }^{48}$ Viger-Gravel, J., A. Schantz, A. C. Pinon, A. J. Rossini, S. Schantz, and L. Emsley. Structure of lipid nanoparticles containing siRNA or mRNA by dynamic nuclear polarization-enhanced NMR spectroscopy. J. Phys. Chem. B 122:2073-2081, 2018.

${ }^{49}$ Vogel, G., and W. Eichenberger. Betaine lipids in lower plants. Biosynthesis of DGTS and DGTA in Ochromonas danica (Chrysophyceae) and the possible role of DGTS in LIPID METABOLISM. Plant Cell Physiol. 33:427-436, 1992.

${ }^{50}$ Yanez Arteta, M., et al. Successful reprogramming of cellular protein production through mRNA delivered by functionalized lipid nanoparticles. Proc. Natl. Acad. Sci. 115:E3351-E3360, 2018.

${ }^{51}$ Yin, H., et al. Structure-guided chemical modification of guide RNA enables potent non-viral in vivo genome editing. Nat. Biotechnol. 35:1179-1187, 2017.

Publisher's Note Springer Nature remains neutral with regard to jurisdictional claims in published maps and institutional affiliations. 